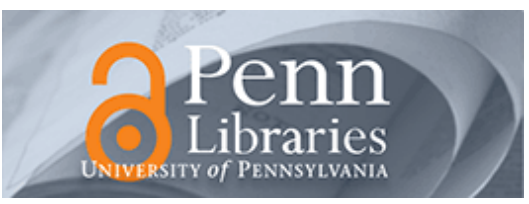

University of Pennsylvania

ScholarlyCommons

December 1996

\title{
Electrostatic "Fractional" Image Methods for Perfectly Conducting Wedges and Cones
}

\author{
Nader Engheta \\ University of Pennsylvania, engheta@ee.upenn.edu
}

Follow this and additional works at: https://repository.upenn.edu/ese_papers

\begin{abstract}
Recommended Citation
Nader Engheta, "Electrostatic "Fractional" Image Methods for Perfectly Conducting Wedges and Cones", . December 1996.
\end{abstract}

Copyright 1996 IEEE. Reprinted from IEEE Transactions on Antennas and Propagation, Volume 44, Issue 12, December 1996, pages 1565-1574.

This material is posted here with permission of the IEEE. Such permission of the IEEE does not in any way imply IEEE endorsement of any of the University of Pennsylvania's products or services. Internal or personal use of this material is permitted. However, permission to reprint/republish this material for advertising or promotional purposes or for creating new collective works for resale or redistribution must be obtained from the IEEE by writing to pubs-permissions@ieee.org. By choosing to view this document, you agree to all provisions of the copyright laws protecting it.

This paper is posted at ScholarlyCommons. https://repository.upenn.edu/ese_papers/243

For more information, please contact repository@pobox.upenn.edu. 


\title{
Electrostatic "Fractional" Image Methods for Perfectly Conducting Wedges and Cones
}

\author{
Abstract \\ In our earlier work, we introduced a definition for the electric charge "fractional-order" multipoles using the \\ concept of fractional derivatives and integrals [1]. Here, we utilize that definition to introduce a detailed \\ image theory for the two-dimensional (2-D) electrostatic potential distributions in front of a perfectly \\ conducting wedge with arbitrary wedge angles, and for the three-dimensional potential in front of a \\ perfectly conducting cone with arbitrary cone angles. We show that the potentials in the presence of \\ these structures can be described equivalently as the electrostatic potentials of sets of equivalent \\ "image" charge distributions that effectively behave as "fractional-order" multipoles; hence, the name \\ "fractional" image methods. The fractional orders of these so-called fractional images depend on the \\ wedge angle (for the wedge problem) and on the cone angle (for the cone problem). Special cases where \\ these fractional images behave like the discrete images are discussed, and physical justification and \\ insights into these results are given.

\section{Comments} \\ Copyright 1996 IEEE. Reprinted from IEEE Transactions on Antennas and Propagation, Volume 44, Issue \\ 12, December 1996, pages 1565-1574. \\ This material is posted here with permission of the IEEE. Such permission of the IEEE does not in any way \\ imply IEEE endorsement of any of the University of Pennsylvania's products or services. Internal or \\ personal use of this material is permitted. However, permission to reprint/republish this material for \\ advertising or promotional purposes or for creating new collective works for resale or redistribution must \\ be obtained from the IEEE by writing to pubs-permissions@ieee.org. By choosing to view this document, \\ you agree to all provisions of the copyright laws protecting it.
}




\title{
Electrostatic "Fractional" Image Methods for Perfectly Conducting Wedges and Cones
}

\author{
Nader Engheta, Fellow, IEEE
}

\begin{abstract}
In our earlier work, we introduced a definition for the electric charge "fractional-order" multipoles using the concept of fractional derivatives and integrals [1]. Here, we utilize that definition to introduce a detailed image theory for the two-dimensional (2-D) electrostatic potential distributions in front of a perfectly conducting wedge with arbitrary wedge angles, and for the three-dimensional potential in front of a perfectly conducting cone with arbitrary cone angles. We show that the potentials in the presence of these structures can be described equivalently as the electrostatic potentials of sets of equivalent "image" charge distributions that effectively behave as "fractional-order" multipoles; hence, the name "fractional" image methods. The fractional orders of these so-called fractional images depend on the wedge angle (for the wedge problem) and on the cone angle (for the cone problem). Special cases where these fractional images behave like the discrete images are discussed, and physical justification and insights into these results are given.
\end{abstract}

\section{INTRODUCTION}

$\mathbf{R}$ ECENTLY, we used the concept of fractional derivatives and integrals to introduce a definition for the "fractionalorder" multipoles in electromagnetism [1]. We showed that for the electrostatic case, as far as their scalar potential distributions are concerned, these fractional-order poles behave effectively as "intermediate" cases between the known integer-order multipoles [1]. In that work, we also alluded that the potentials of the electrostatic fractional-order poles resemble one of the solutions of the Laplace equation for the regions in front of the perfectly conducting cones (for three-dimensional (3-D) case) and for the perfectly conducing wedges (for the two-dimensional (2-D) case). Here, we expand that observation to develop an electrostatic image theory for the 2-D perfectly conducting wedges and for the 3-D perfectly conducting cones using the concept of fractional calculus and fractional-order poles. This idea of electrostatic "fractional" image method was first presented verbally by the author in a recent symposium [2]. Some other applications of fractional calculus in electromagnetic theory have been studied by the author [3], [4]. It should be mentioned that the topic of fractional calculus has found applications in various fields of science (e.g., [5]-[7]).

The classic method of images is a well-known technique often used in electromagnetics to describe the electric potential and field distributions of a given source situated in proximity of objects with certain geometries and material properties (e.g.,

Manuscript received November 16, 1995; revised March 15, 1996.

The author is with the Moore School of Electrical Engineering, University of Pennsylvania, Philadelphia, PA 19104 USA.

Publisher Item Identifier S 0018-926X(96)08367-6.
[8, pt. 1 , ch. 11], [9, pp. 68-70, 124-135]). As described in many standard textbooks in electromagnetics, one of the most basic geometries for which the image method has been used is the perfectly conducting or the dielectric half space with the flat interface (e.g., [9]). Another well-known classic case for which the electrostatic image theory has been applied is the potential distribution of a point charge located in front of a perfectly conducting sphere. The image method solution to this problem has been attributed to Lord Kelvin in 1848 [8, p. 245]. As mentioned by Lindell et al. [10], the dielectric counterpart of this problem was addressed by Neumann (in 1883), and also treated by Lindell (in 1992) [11]. For an excellent treatment of some of the classical image methods, the reader is referred to Smythe [9].

The method of images for the perfectly conducting half space (or equivalently for a perfectly conducting flat plate) is straightforwardly extended to the 2-D perfectly conducting corners (i.e., intersecting plates) with specific outer angles $[9$, p. 70$]$. Specifically, it is known that if an infinitely long uniform electrostatic line charge is located parallel with the edge of the corner in front of a perfectly conducting corner, and if the corner's outer angle denoted by $\beta$ is such that $\pi / \beta$ is an integer, then there exist $(2 \pi / \beta-1)$ discrete images $[9$, p. 70$]$. Thus, the electrostatic potentials due to the original line charge together with these $(2 \pi / \beta-1)$ images can provide the potential distribution in front of the perfectly conducting corner. An obvious question to ask here is: what kind of "images" (if any) would one get if $\pi / \beta$ were not an integer? Would we get a distributed set of images? One answer to these questions can be obtained from the elegant work of Nikoskinen and Lindell who have analyzed the problem of image solutions for the Poisson equation for the dielectric wedge geometry [12]. They found that for the perfectly conducting wedge with an arbitrary angle, their image solutions have a distributed portion in the imaginary angular domain [12]. Lindell and his co-workers have also treated some other interesting cases such as 2-D electromagnetic image theory for conducting wedges [13] and conducting half planes [14] and transient problems [15], [16].

In this paper, we use a completely different approach to treat the problem of 2-D electrostatic image problems for the perfectly conducting wedge with arbitrary angles. We will show that for this problem, using the concept of "fractionalorder" multipoles [1], it is possible to describe "images" that effectively behave as distributed "intermediate" cases between those discrete images obtained for specific wedge angles. The fractional orders of these equivalent "images," which we call "fractional" images, depend on the wedge's angle. We also 


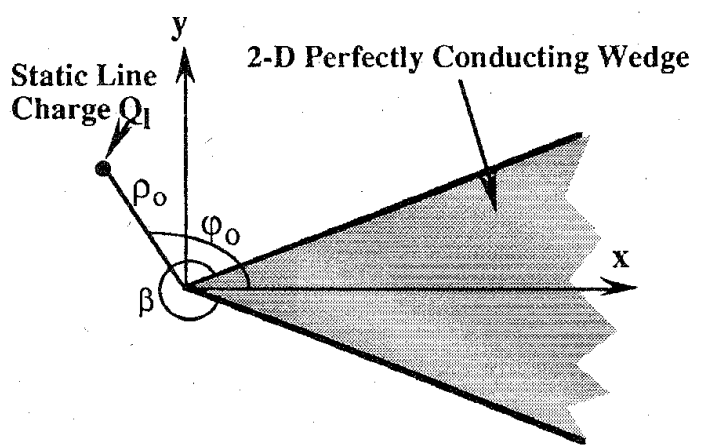

Fig. 1. Two-dimensional perfectly conducting wedge with the outer angle $\beta$ An infinitely long static line charge with uniform line charge density of $Q_{l}$ (Coulomb/m) is located at $\left(\rho_{0}, \varphi_{0}\right)$. The Cartesian $(x, y, z)$ and cylindrical $(\rho, \varphi, z)$ coordinate systems are used. The geometry of the problem is independent of $z$

extend this image method for the 3-D perfectly conducting cones.

\section{Two-Dimensional Electrostatic Image Methods For PERfeCtLy CONDUCTING Ẇedges WITH ARBITRARY ANGLES}

Consider a 2-D perfectly conducting wedge with the outer angle of $\beta$. A Cartesian coordinate system $(x, y, z)$ is chosen with the $z$ axis along the edge of the wedge, and the $x-z$ plane being the symmetry plane of the wedge. (Fig. 1). A cylindrical coordinate system $(\rho, \varphi, z)$ is also used with $x=\rho \cos \varphi$ and $y=\rho \sin \varphi$. An infinitely long uniform static line charge with charge density per unit length of $Q_{l}$ (Coulomb/m) is located parallel with the $z$ axis at an arbitrary point with coordinates $\left(\rho_{0}, \varphi_{0}\right)$. The electrostatic potential $\Phi_{l w}(\rho, \varphi)$ due to this line charge in front of the perfectly conducting wedge (i.e., in the angular range of $\pi-\beta / 2<\varphi<\pi+\beta / 2$ ); can be written as

$$
\begin{aligned}
\Phi_{l w}(\rho, \varphi)= & \sum_{m=1}^{\infty} \frac{Q_{l}}{m \pi \varepsilon}\left(\frac{\rho_{<}}{\rho_{>}}\right)^{m \pi / \beta} \\
& \cdot \sin \left[\frac{m \pi}{2}-\frac{m \pi}{\beta}(\pi-\varphi)\right] \\
& \cdot \sin \left[\frac{m \pi}{2}-\frac{m \pi}{\beta}\left(\pi-\varphi_{0}\right)\right]
\end{aligned}
$$

where $\rho_{<}\left(\rho_{>}\right)$is the smaller (larger) of $\rho_{0}$ and $\rho$, respectively, $\epsilon$ is the permittivity of the homogeneous isotropic medium in front of the wedge $[17, \mathrm{p} .76]$. Now let us consider the case of the line charge alone (without the wedge) placed at the same location $\left(\rho_{0}, \varphi_{0}\right)$. The electrostatic potential of this uniform line charge can be expressed [17, p. 118] as

$$
\begin{gathered}
\Phi_{l o}(\rho, \varphi)=C-\frac{Q_{l}}{2 \pi \varepsilon} \ln \left(\rho_{>}\right) \\
+\sum_{m=1}^{\infty}\left\{\frac { Q _ { l } } { 2 m \pi \varepsilon } ( \frac { \rho _ { < } } { \rho _ { > } } ) ^ { m } \left[\cos \left(m \varphi_{0}\right) \cos (m \varphi)\right.\right. \\
\left.\left.+\sin \left(m \varphi_{0}\right) \sin (m \varphi)\right]\right\}
\end{gathered}
$$

where the constant $C$ (which is unimportant in our discussion here) is the usual arbitrary constant to be chosen such that the potential acquires a specific value at a given point. Let us consider the region where $\rho>\rho_{0}$. For this region, the expansion of $\Phi_{l o}(\rho, \varphi)$ in terms of 2-D circular harmonics reveals the role and importance of each term. Specifically, it is known that $(\ln \rho)$ term is due to the line monopole contribution, the terms $\rho^{-1} \cos (\varphi)$ and $\rho^{-1} \sin (\varphi)$ are due to the line dipole contributions, the terms $\rho^{-2} \cos (2 \varphi)$ and $\rho^{-2} \sin (2 \varphi)$ are due to the line quadrupole contributions, etc. It is also interesting to note that one can write

$$
\left\{\begin{array}{l}
\cos (\varphi) \\
\sin (\varphi)
\end{array}\right\} \frac{1}{\rho}=\left\{\begin{array}{l}
\frac{\partial}{\partial x} \\
\frac{\partial}{\partial y}
\end{array}\right\}[\ln (\rho)]
$$

and one can extend this feature to the higher-order terms with $m \geq 1$ as follows ${ }^{1,2}$

$$
\begin{aligned}
\left\{\begin{array}{c}
\cos (m \varphi) \\
\sin (m \varphi)
\end{array}\right\} \frac{1}{\rho^{m}}= & \frac{(-1)^{m-1}}{(m-1) !} \frac{\partial^{m-1}}{\partial x^{m-1}}\left\{\left\{\begin{array}{l}
\cos (\varphi) \\
\sin (\varphi)
\end{array}\right\} \frac{1}{\rho}\right\} \\
= & \frac{(-1)^{m}}{(m-1) !}\left\{\begin{array}{c}
\frac{\partial^{m}}{\partial x^{m}} \\
\frac{\partial^{m}}{\partial x^{m-1} \partial y}
\end{array}\right\} \\
& \cdot[-\ln (\rho)] .
\end{aligned}
$$

When we substitute (4) into (2) for the case where $\rho>\rho_{0}$, we obtain

$$
\begin{aligned}
\Phi_{l o}(\rho, \varphi)=\frac{Q_{l}}{2 \pi \varepsilon} \sum_{m=0}^{\infty}\{ & \frac{(-1)^{m} \rho_{0}^{m}}{m !}\left[\cos \left(m \varphi_{0}\right) \frac{\partial^{m}}{\partial x^{m}}\right. \\
& \left.\left.+\sin \left(m \varphi_{0}\right) \frac{\partial^{m}}{\partial x^{m-1} \partial y}\right]\left[C^{\prime}-\ln (\rho)\right]\right\}
\end{aligned}
$$$$
\text { for } \rho>\rho_{0}
$$

where $C^{\prime}\left[\equiv\left(2 \pi \varepsilon / Q_{l}\right) C\right]$ is a constant $(m=0$ term is included). From (5), it can be seen that for an observer in the region $\rho>\rho_{0}$ an equivalent representation for a 2-D charge distribution, which would provide the same potential as that of the single line charge (in the absence of the wedge), can be written as

$$
\begin{aligned}
Q_{\text {equiv }}=Q_{l} \delta(x) \delta(y) \\
+\sum_{m=1}^{\infty}\left\{\frac{\rho_{0}^{m} \cos \left[m\left(\pi-\varphi_{0}\right)\right]}{\Gamma(m+1)}\left[Q_{l} \delta^{(m)}(x) \delta(y)\right]\right\} \\
+\sum_{m=1}^{\infty}\left\{\frac{\rho_{0}^{m} \sin \left[m\left(\pi-\varphi_{0}\right)\right]}{\Gamma(m+1)}\right. \\
\left.\cdot\left[-Q_{l} \delta^{(m-1)}(x) \delta^{(1)}(y)\right]\right\}
\end{aligned}
$$

\footnotetext{
${ }^{1}$ For the 3-D case, Maxwell has shown that the spherical harmonics can be written in terms of multiple spatial differentiations of potential of a point charge [8, pp. 207-214].

${ }^{2}$ One can show that the representation of $\rho^{-m} \cos (m \varphi)$ and $\rho^{-m} \sin (m \varphi)$ in terms of the spatial derivatives of $-\ln (\rho)$ can have a more general form. It can be shown that

and

$$
\rho^{-m} \cos (m \varphi)=\frac{(-1)^{m} \partial^{m}}{(m-1) ! \partial x^{m-4 s} \partial y^{4 s}}[-\ln (\rho)]
$$

$$
\rho^{-m} \sin (m \varphi)=\frac{(-1)^{m} \partial^{m}}{(m-1) ! \partial x^{m-4 s-1} \partial y^{4 s+1}}[-\ln (\rho)]
$$

where $s=0,1,2,3, \cdots$ Here, in (4), $s$ is taken to be zero. Furthermore, the technique shown by Maxwell [8, pp. 207-214] can be used with appropriate modification for the 2-D case.
} 
where $\delta^{(m)}(\cdot)$ denotes the $m$ th-order derivative of the Dirac delta function ${ }^{3}$ and the identity $\Gamma(m+1)=m$ ! has been used. For the reason that will become clear later, the terms $(-1)^{m}$ have been combined with the $\cos (\cdot)$ and $\sin (\cdot)$ terms in the above expression. This description is consistent with the concept of multipole expansion of electric sources [20, ch. 4], [21]-[23] and with the mathematical forms of charge density distributions for point multipoles [21]-[23] (or line multipoles for the 2-D case). The expression in (6) emphasizes the fact that, aside from the specific multiplicative constants, the shape of the spatial distributions of the higher-order line multipoles can be written in terms of spatial derivatives of Dirac delta functions. As will become apparent shortly, we will utilize and expand this concept to introduce the concept of "fractional" images as equivalent representation for a charge distribution for the potentials involving perfectly conducting wedges.

Returning to the case of the line charge in front of the perfectly conducting wedge shown in Fig. (1), one can see from (1) (for $\rho>\rho_{0}$ ) that the exponents of the $\rho$-dependences of various terms of the potential involve $-(m \pi / \beta)$ which are, in general, nonintegers. However, it is well known that for a 2-D static electric charge density with its cross section in the $x-y$ plane bounded in a finite region (and with no wedge present), the multipole expansion of the potential involves terms whose $\rho$-dependences have negative integer exponents (except the zeroth-order line monopole whose potential has logarithmic dependence on $\rho$ ). Therefore, because of the noninteger exponents $m \pi / \beta$ one can conclude that for the wedge problem, shown in Fig. 1, an equivalent representation of charge distribution which would provide a potential distribution similar to that of the wedge in the region $\rho>\rho_{0}$ will not be limited in a certain bounded region in the 2D $x-y$ plane (i.e., it would not be localized in the $x-y$ plane). In other words, for the noninteger $\pi / \beta$, any image charges (or portion thereof) for the potential problem involving perfectly conducting wedge with outer angle $\beta$ will be of the nonlocalized distributed form (instead of being discrete image charges). ${ }^{4}$

Let us consider the dominant term in (1) for $\rho>\rho_{0}$. The $\rho$-dependence of this term has the form $\rho^{-\pi / \beta}$ which decays faster than $-\ln (\rho)$ term (of a line monopole) and decays more slowly than $\rho^{-n}$ (of a line $2^{n}$-pole) where $n$ is the first positive integer larger than $\pi / \beta$. Therefore, for $\mathrm{B}>\pi$ it appears that in the absence of the wedge (and the original line charge), an equivalent (or substituting) charge distribution to produce a potential with $\rho^{-\pi / \beta}$ dependence should have a form which is neither a line monopole nor a line dipole at the origin, instead it should effectively be an "intermediate" case between a 2-D line monopole and a 2-D line dipole. This can be a case to utilize the concept of "fractional-order" multipoles.

\footnotetext{
${ }^{3}$ In the rigorous mathematical sense, the Dirac delta function should be treated as a generalized function, and its derivatives should also be handled in that context [18], [19].

${ }^{4}$ In fact, in the presence of the wedge it is obvious that the part of the actual charge densities involved in the wedge problem, viz., the charge induced on the surface of the perfectly conducting wedge, is not localized in a certain bounded region in the $x-y$ plane. Instead, it is distributed on the surface of the wedge extending to infinity.
}

We showed elsewhere [1] that a 2-D "fractional-order" multipole (e.g., $2^{\alpha}$-pole with $0<\alpha<1$ ) can be obtained by taking the $\alpha$ th-order fractional derivative of the 2-D Dirac delta function $\delta(x) \delta(y)$. The 2-D charge density distribution of such a $2^{\alpha}$ pole can be written as

$$
\begin{aligned}
Q_{2^{\alpha}, x}(x, y) & =l^{\alpha}{ }_{-\infty} D_{x}^{\alpha}\left[Q_{l} \delta(x) \delta(y)\right] \\
& =Q_{l} l^{\alpha} \frac{\partial^{2}}{\partial x^{2}} \begin{cases}\delta(y) \frac{1}{\Gamma(2-\alpha)} x^{1-\alpha} & \text { for } x>0 \\
0 & \text { for } x<0\end{cases}
\end{aligned}
$$

where $Q_{2^{\alpha}, x}(x, y)$ denotes the volume charge density (unit of Coulomb $/ \mathrm{m}^{3}$ ) of this 2-D charge distribution, the subscript $2^{\alpha}$ with noninteger $\alpha$ indicates the fractional order of the multipole (e.g., for $\alpha=0$ we have a monopole, and for $\alpha=1$ we have a dipole), subscript $x$ shows that the fractional derivative is taken with respect to the $x$ coordinate, $Q_{l}$ (with unit of Coulomb/m) is the strength of the static line charge (line monopole at $x=y=0$ ), $-\infty D_{x}^{\alpha}$ is the symbol for the fractional derivative of order $\alpha$ with respect to variable $x$ and with the lower limit of $-\infty . .^{5}$ The arbitrary multiplicative constant $l^{\alpha}$, with $l$ being a constant with dimension of length, is used here to keep the physical dimensions of quantities unchanged when a fractional derivative is taken. Therefore, when we take $\alpha$ th-order $x$-derivative of $Q_{l} \delta(x) \delta(y)$, the multiplicative constant $l^{\alpha}$ keeps the dimension of $Q_{2^{\alpha}, x}(x, y)$ as Coulomb $/ \mathrm{m}^{3}$. The sketch of such a charge distribution is shown in Fig. 2(a). We have analyzed the electrostatic potential distributions and field components of such a charge distribution elsewhere [1]. The details are not repeated here and the reader is referred to our previous work [1]. The potential of such a charge distribution can be expressed in a closed form as

$$
\Phi_{2^{\alpha}, x}(\rho, \varphi)= \begin{cases}\frac{Q_{l} l^{\alpha} \Gamma(\alpha)}{2 \pi \varepsilon \rho^{\alpha}} \cos [\alpha(\pi-\varphi)] & \text { for } 0<\alpha \\ C-\frac{Q_{l}}{2 \pi \varepsilon} \ln (\rho) & \text { for } \alpha=0\end{cases}
$$

for $\rho>0$ and $0<\varphi<2 \pi$. It can be easily shown that the above expressions for the electrostatic potential (and corresponding field components [1]) of the $2^{\alpha}$ pole approach their corresponding limiting values for the infinitely long line charge (limit of $\alpha \rightarrow 0$ ) and those of the line dipole located parallel with the $z$ axis and the dipole vector along the $x$ axis

${ }^{5}$ There are several definitions for the fractional integrals and derivatives [24]-[26]. One of the definitions of fractional integral is the Riemann-Liouville integral [24, p. 49]. For a given function $f(x)$, the Riemann-Liouville integral for the fractional integral is defined as

$$
\begin{gathered}
{ }_{a} D_{x}^{\alpha} f(x) \equiv \frac{1}{\Gamma(-\alpha)} \int_{a}^{x}(x-u)^{-\alpha-1} f(u) d u \\
\text { for } \alpha<0 \text { and } x>a .
\end{gathered}
$$

For cases where $\alpha$ becomes a negative integer, this integral becomes Cauchy's repeated integration $\left[24\right.$, p. 38]. (Here, in this paper the notation ${ }_{a} D_{x}^{\alpha}$, developed by Davis [25], has been adapted.) For fractional derivatives where $\alpha>0$, the Riemann-Liouville integral can be used if one uses the additional step ${ }_{a} D_{x}^{\alpha} f(x)=\left(d^{m} / d x^{m}\right)_{a} D_{x}^{\alpha-m} f(x)$, where $m$ is chosen such that $(\alpha-m)$ becomes negative, and thus, the above integration can be applied for ${ }_{a} D_{x}^{\alpha-m} f(x)$. Then $d^{m} / d x^{m}$ is the conventional $m$ th-order differential operator $[24$, p. 50]. It can be shown that when $\alpha$ is a positive integer, the fractional derivative results in the conventional derivatives. 
(limit of $\alpha \rightarrow 1)^{6}$ As an illustrative example, the contour plot of (8) is given in Fig. 2(b) for a fractional value of $\alpha=0.8$. If we compare the potential given in (8) for $\alpha>0$ with one of the terms in (1) of the wedge problem (for $\rho>\rho_{0}$ ), we will notice that for the terms with $m=$ odd integer, the two expressions will become similar when $\alpha$ takes the value of $m \pi / \beta$. With the proper choice of a multiplicative constant, these two terms can become the same. Therefore, if we introduce a multiplicative constant $C_{\alpha}$ in front of the charge density distribution of the fractional $2^{\alpha}$-pole, this constant can be chosen such that the electrostatic potential of the fractional $2^{\alpha}$-pole becomes the same as one of the odd terms in (1). In other words, we can show that the potential given in (8) becomes the same as the potential of the $m=(2 n+1)$ st term in (1) for the wedge problem (for $\rho>\rho_{0}$ ), when $\alpha=m \pi / \beta=(2 n+1) \pi / \beta$ with $n=0,1,2,3, \cdots$ and

$$
\begin{aligned}
& C_{\alpha=(2 n+1) \pi / \beta}= \\
& \frac{2 \pi \cos \left[\frac{(2 n+1) \pi}{\beta}\left(\pi-\varphi_{0}\right)\right]\left(\frac{\rho_{0}}{l}\right)^{(2 n+1) \pi / \beta}}{\beta \Gamma\left[\frac{(2 n+1) \pi}{\beta}+1\right]} \\
& \text { with } n=0,1,2, \cdots .
\end{aligned}
$$

Therefore, the "fractional-order" $2^{(2 n+1) \pi / \beta}$-pole is described as follows:

$$
\begin{aligned}
& Q_{\text {equiv }}(x, y)_{\text {for } m=2 n+1} \\
& =C_{(2 n+1) \pi / \beta} l_{-\infty}^{\alpha} D_{x}^{(2 n+1) \pi / \beta}\left[Q_{l} \delta(x) \delta(y)\right] \\
& =\frac{2 \pi \rho_{0}^{(2 n+1) \pi / \beta} \cos \left[\frac{(2 n+1) \pi}{\beta}\left(\pi-\varphi_{0}\right)\right]}{\beta \Gamma\left[\frac{(2 n+1) \pi}{\beta}+1\right]} \\
& \cdot{ }_{-\infty} D_{x}^{(2 n+1) \pi / \beta}\left[Q_{l} \delta(x) \delta(y)\right]
\end{aligned}
$$

can be regarded as an equivalent charge representation for the terms with $m=(2 n+1)$ in the expression of potential of the wedge for the region $\rho>\rho_{0}$. Thus, in the absence of the wedge and the original line charge this fractional-order pole, which involves fractional derivative of the line charge at the origin, produces the $(2 n+1)$ st term of the wedge potential in the region $\rho>\rho_{0}$. The order of the fractional derivative is $(2 n+1) \pi / \beta$, which is directly dependent on the wedge's outer angle.

For the terms with even numbers $m=2 n+2$ in (1) with $\rho>\rho_{0}$, we need to look at another form of fractional-order multipole. We can show that if instead of taking fractional $\alpha$ th-order derivative of the line monopole with respect to $x$ we take first-order derivative of the line monopole with respect to $y$, and then take fractional $(\alpha-1)$ th-order derivative with respect to $x$, we will obtain certain charge distribution whose potentials will resemble the terms with $m=2 n+2$ in (1). More specifically, we define the following fractional-order

\footnotetext{
${ }^{6}$ It can also be shown that for $\alpha=n$ (integers), these expressions can also approach their corresponding values for higher integer-order line multipoles with multipole tensor components along the $x$ axis.
}

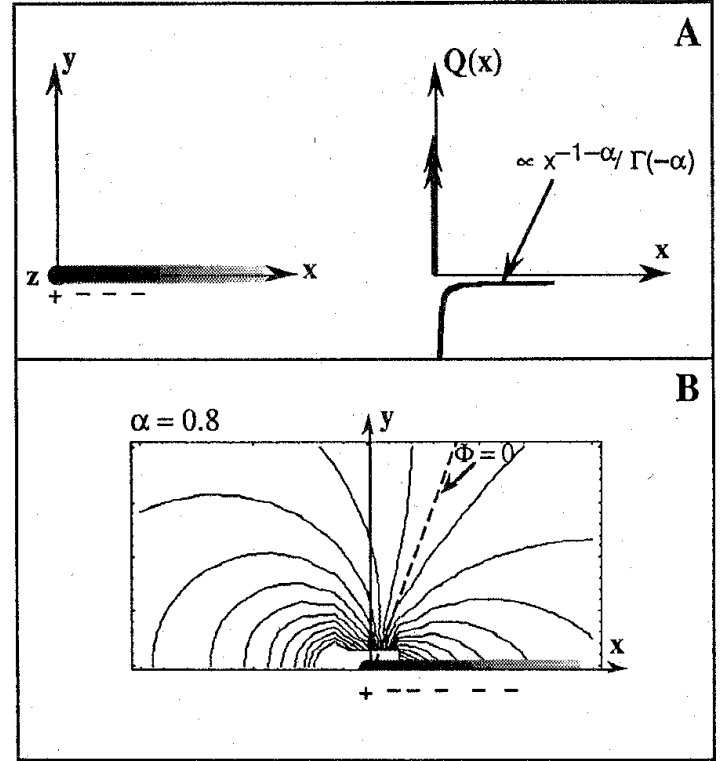

Fig. 2. In the left side of panel $A$, the sketch of a fractional $2 \alpha$-pole in the 2-D case is shown with $0<\alpha<1$. (The $\alpha$ th-order fractional derivative is taken with respect to $x$ ). In the right side, the form of the volume charge density for the charge distribution shown in left, is illustrated. This volume charge density, which is a surface charge distribution in the $x-z$ plane, has the form of $Q(x) \delta(y)$, where the form of the function $Q(x)$ as a function of $x$ is only shown. (Here, for purpose of plotting $Q(x)$ for $x>0, \alpha$ is taken to be $\alpha=0.8$.) At $x=0$, the expression for (7) has a singularity in the form resulting from $\left(\partial^{2} / \partial x^{2}\right)\left[U(x) x^{1-\alpha} / \Gamma(2-\alpha)\right]$ at $x=0$ where $U(x)$ is the unit-step function. In panel $B$, contour plots are shown for the 2-D electrostatic scalar potential of the fractional $2^{\alpha}$ pole shown in panel $\mathrm{A}$. These contours are drawn using the computer program Mathematica ${ }^{3}$ (Version 2.0, Wolfram Research, Inc.). For plotting purposes, the expression of potential in (8) is normalized with $Q_{l} l^{\alpha} /(2 \pi \varepsilon)=1$. The intersection of equipotential surfaces with the $x-y$ plane is shown here. Since the potential distribution is symmetric with respect to the $x-z$ plane, the contours are only shown for the region $-2<x<+2$ and $0<y<2$. The parameter $\alpha=0.8$ is used for the plot. The potential to the left of the surface $\Phi=0$ is positive.

multipole:

$$
\begin{aligned}
& Q_{2^{\alpha-1}, x ; 2^{1}, y}(x, y) \\
& \quad=C_{\alpha} l_{x}^{\alpha-1} l_{y-\infty} D_{x}^{\alpha-1}\left\{\frac{\partial}{\partial y}\left[Q_{l} \delta(x) \delta(y)\right]\right\} \\
& =-C_{\alpha} l_{x}^{\alpha-1}-\infty D_{x}^{\alpha-1}\left[-Q_{l} l_{y} \delta(x) \delta^{(1)}(y)\right]
\end{aligned}
$$

where $C_{\alpha}$ is a constant to be determined such that the potential of this fractional-order multipole becomes the same as a term with $m=2 n+2$ in (1), $l_{x}^{\alpha-1}$ and $l_{y}$ are also two multiplicative constants with $l_{x}$ and $l_{y}$ being two constants with dimension of length to assure the proper physical dimensions for the charge densities of the fractional-order multipole. The term in the last square brackets in (11) represents a line dipole at the origin with lines being parallel with the $z$ axis and with its dipole vector in the $y$ direction. Thus, such a fractional-order multipole can be interpreted as the fractional $(\alpha-1)$ th-order derivative (with respect to $x$ ) of that line dipole. In contrast, the fractional-order multipole given in (7) was the fractional $\alpha$ th-order derivative (with respect to $x$ ) of the line monopole at the origin. The sketch of the charge distribution in (11) is shown in Fig. 3(A). The electrostatic potential of such a 


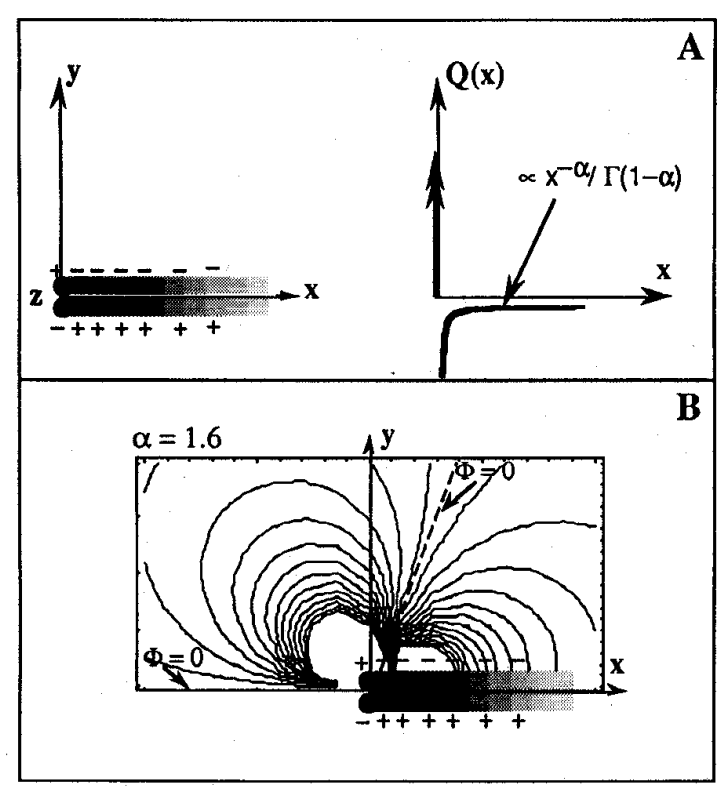

Fig. 3. Similar description, as in caption of Fig. 2, except here we have the fractional-order pole which is obtained by taking fractional $(\alpha-1)$ th-order derivative of a line dipole $\left[-Q_{l} \delta(x) \delta^{(1)}(y)\right]$ [see (11)]. For plotting purposes, $\alpha$ is taken to be 1.6. The potential distribution in this case is antisymmetric with respect to the $x-z$ plane. The potential between the two planes of $\Phi=0$, shown above, is positive.

fractional-order multipole can be explicitly written as

$$
\begin{aligned}
\Phi_{2^{\alpha-1}, x ; 2^{1}, y}(\rho, \varphi)=- & C_{\alpha} \frac{Q_{l} l_{x}^{\alpha-1} l_{y} \Gamma(\alpha)}{2 \pi \varepsilon \rho^{\alpha}} \sin [\alpha(\pi-\varphi)] \\
& \text { for } 0<\alpha
\end{aligned}
$$

for $\rho>0$ and $0<\varphi<2 \pi$, and a contour plot of this potential is depicted in Fig. 3(B) for $\alpha=1.6$. Comparing this potential with terms with $m=2 n+2$ in (1) for the wedge potential for $\rho>\rho_{0}$, we observe that for $\alpha=(2 n+2) \pi / \beta$ with $n=0,1,2, \cdots$, and

$$
\begin{aligned}
& C_{\alpha=(2 n+2) \pi / \beta}= \\
& \frac{-2 \pi \sin \left[\frac{(2 n+2) \pi}{\beta}\left(\pi-\varphi_{0}\right)\right] \frac{\rho_{0}^{(2 n+2) \pi / \beta}}{l_{x}^{(2 n+2) \pi / \beta-1} l_{y}}}{\beta \Gamma\left[\frac{(2 n+2) \pi}{\beta}+1\right]} \\
& \text { with } n=0,1,2, \cdots
\end{aligned}
$$

the two potentials become the same. Therefore, in the absence of the wedge and the original line charge, the fractional-order $2^{(2 n+2) \pi / \beta}$-pole, described as

$$
\begin{aligned}
Q_{\text {equiv }}(x, y)_{\text {for }} \quad \begin{array}{l}
m=2 n+2 \\
=
\end{array} & C_{(2 n+2) \pi / \beta} l_{x}^{(2 n+2) \pi / \beta-1} \\
& \cdot-\infty D_{x}^{(2 n+2) \pi / \beta-1}\left[Q_{l} l_{y} \delta(x) \delta^{(1)}(y)\right] \\
= & \frac{2 \pi \rho_{0}^{(2 n+2) \pi / \beta} \sin \left[\frac{(2 n+2) \pi}{\beta}\left(\pi-\varphi_{0}\right)\right]}{\beta \Gamma\left[\frac{(2 n+2) \pi}{\beta}+1\right]} \\
& \cdot-\infty D_{x}^{(2 n+2) \pi / \beta-1}\left[-Q_{l} \delta(x) \delta^{(1)}(y)\right]
\end{aligned}
$$

which involves fractional $[(2 n+2) \pi / \beta-1]$ th-derivative (with respect to $x$ ) of the line dipole, will produce, in the region $\rho>\rho_{0}$, a potential similar to the $(2 n+2)$ nd term for the wedge potential. Given the fractional-order multipoles described above for the even- $m$ and odd- $m$ terms in the wedge potential expression for the region $\rho>\rho_{0}$, one can write [in the absence of the wedge and its original line charge at $\left.\left(\rho_{0}, \varphi_{0}\right)\right]$ the following expression as the combined equivalent charges that would produce the same potential for the region $\rho>\rho_{0}$

$$
\begin{aligned}
& \sum_{n=0}^{Q_{\text {equiv }}(x, y)=}\left\{\frac{2 \pi \rho_{0}^{(2 n+1) \pi / \beta} \cos \left[\frac{(2 n+1) \pi}{\beta}\left(\pi-\varphi_{0}\right)\right]}{\beta \Gamma\left[\frac{(2 n+1) \pi}{\beta}+1\right]}\right. \\
& +\sum_{n=0}^{\infty}\left\{\frac{\left.-\infty D_{x}^{(2 n+1) \pi / \beta}\left[Q_{l} \delta(x) \delta(y)\right]\right\}}{\beta \Gamma\left[\frac{(2 n+2) \pi}{\beta}+1\right]} \rho_{0}^{(2 n+2) \pi / \beta} \sin \left[\frac{(2 n+2) \pi}{\beta}\left(\pi-\varphi_{0}\right)\right]\right. \\
& \left.\cdot-\infty D_{x}^{(2 n+2) \pi / \beta-1}\left[-Q_{l} \delta(x) \delta^{(1)}(y)\right]\right\}
\end{aligned}
$$

It is interesting to compare and/or contrast the above combined charge distribution, with (6) which is the equivalent representation of charge distribution for a single line charge located at $\left(\rho_{0} \varphi_{0}\right)$ (without any wedge present). First, we notice that (6) has a line monopole term $Q_{l} \delta(x) \delta(y)$, whereas (15) does not. This is due to the fact that for a single line-charge problem, the two-dimensional total charge per unit length, is not zero; hence, the nonzero monopole term. However, in the perfectly conducting wedge problem with the line charge in its proximity, considering the line charge and the induced charge on the surfaces of the perfectly conducting wedge, the total net charge per unit length (along $z$ ) is zero; thus, there should not be any line-monopole term. Second, the mathematical forms of the two equations are similar in that both involve derivatives of the line monopole $\left[Q_{l} \delta(x) \delta(y)\right]$ and the line dipole $\left[-Q_{l} \delta(x) \delta^{(1)}(y)\right]$. However, in (6), conventional derivatives of order $m$ and $m-1$ are involved, whereas in (15) fractional derivatives of orders $[(2 n+1) \pi / \beta]$ and $[(2 n+2) \pi / \beta-1]$ should be employed. It is also worth noting that this differentiation is with respect to the $x$ coordinate, which is the symmetry line in the wedge's cross section with the $x-y$ plane. Third, it must be noted that the multiplicative constants in front of these derivatives have similar forms, with the understanding that the parameter $m$ in (6) is replaces. by $[(2 n+1) \pi / \beta]$ and $[(2 n+2) \pi / \beta-1]$ in (15). Finally, there is a factor of $2 \pi / \beta$ present in (15) which is due to the difference in orthonormalization factors of the orthogonal sinusoidal functions $\sin [m \pi / 2-(m \pi / \beta)(\pi-\varphi)]$ for the 
angular range of $\pi-\beta / 2<\varphi<\pi+\beta / 2$, and the functions $\sin (m \varphi)$ and $\cos (m \varphi)$ for the angular range of $0<\varphi<2 \pi$. To have better understanding of the physical meaning of the equivalent (or substituting) charge given in (15), let us consider the observation points in the far region, i.e., where $\rho \gg \rho_{0}$. For this case, the dominant term in the wedge potential given in (1) is the term with $\rho^{-\pi / \beta}$ dependence. Thus, we have

$$
\begin{aligned}
\Phi_{l w}(\rho, \varphi) \approx & \frac{Q_{l}}{\pi \varepsilon}\left(\frac{\rho_{0}}{\rho}\right)^{\pi / \beta} \cos \left[\frac{\pi}{\beta}\left(\pi-\varphi_{0}\right)\right] \\
& \cdot \cos \left[\frac{\pi}{\beta}(\pi-\varphi)\right] \text { for } \rho \gg \rho_{0} .
\end{aligned}
$$

If we remove the perfectly conducting wedge and the line charge in its proximity, and substitute them with the first term of the charge distribution in (15), viz., $m=$ first term which is

$$
\begin{aligned}
& Q_{\text {equiv }}(x, y) \approx \frac{2 \pi \rho_{0}^{\pi / \beta} \cos \left[\frac{\pi}{\beta}\left(\pi-\varphi_{0}\right)\right]}{\beta \Gamma\left[\frac{\pi}{\beta}+1\right]} \\
& { }_{-\infty} D_{x}^{\pi / \beta}\left[Q_{l} \delta(x) \delta(y)\right] \\
& =\frac{2 \pi Q_{l} \rho_{0}^{\pi / \beta} \cos \left[\frac{\pi}{\beta}\left(\pi-\varphi_{0}\right)\right]}{\beta \Gamma\left[\frac{\pi}{\beta}+1\right]} \frac{\partial^{p}}{\partial x^{p}} \\
& \begin{cases}\delta(y) \frac{1}{\Gamma\left[p-\frac{\pi}{\beta}\right]} x^{-\pi / \beta+p-1} & \text { for } x>0 \\
0 & \text { for } x<0\end{cases}
\end{aligned}
$$

we will obtain the same potential as (16). Here, integer $p$ is chosen such that $\pi / \beta-p$ becomes negative, and thus, the Riemann-Liouville integral for the definition of fractional integral can be used (see footnote 5). At $x=0$, care must be exercised when evaluating $\partial^{p} / \partial x^{p}$. The integer $p$ can be chosen such that the function $x^{-\pi / \beta+p-1}$ becomes continuous at $x=0$. However, $p$ th-order derivative of this function at $x=0$ is singular and discontinuous. For noninteger values of $\pi / \beta$, this 2-D charge density distribution is a (nonlocalized) surface-charge distribution on the $x-z$ plane for $x>0$, with volume charge density given in the above equation. Fig. 4 shows the sketch of this charge distribution, which is to substitute the wedge and its neighboring line charge.

Let us consider the special cases for which $\pi / \beta$ is an integer. It is well known from the conventional image method that for these values, one can find a finite number of discrete (i.e., localized) image charges. If $\beta=\pi$, the wedge becomes a 2-D perfectly conducting half space with flat interface on the $y-z$ plane. For this case, the electrostatic potential for the distant observer (16) is given as

$$
\begin{aligned}
\Phi_{l w}(\rho, \varphi)_{\beta=\pi} \approx Q_{l} \rho_{0} \cos \left(\varphi_{0}\right) \frac{\cos (\varphi)}{\pi \varepsilon \rho} \\
\text { for } \rho \gg \rho_{0} \\
\text { and } \frac{\pi}{2}<\varphi, \varphi_{0}<\frac{3 \pi}{2}
\end{aligned}
$$

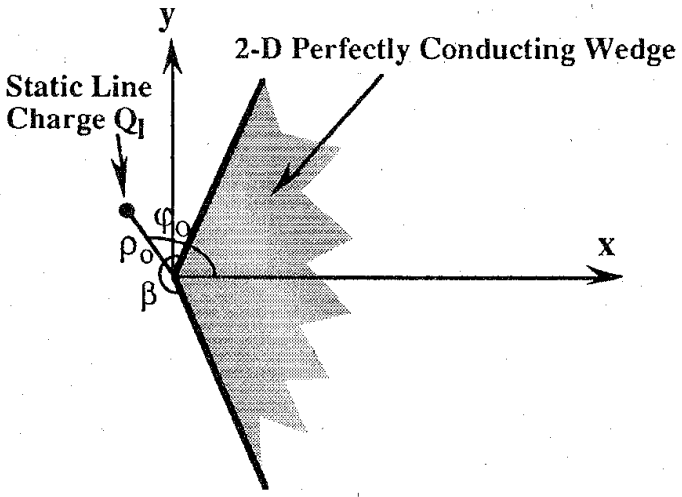

(a)

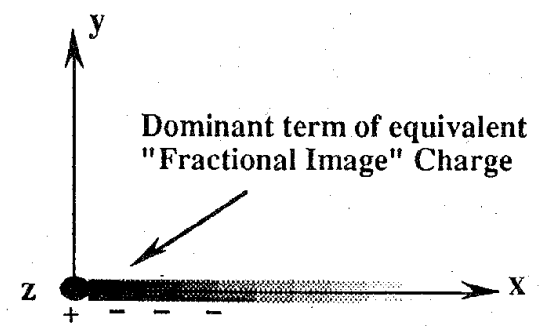

(b)

Fig. 4. (a) A 2-D perfectly conducting wedge with an infinitely long static line charge in front of it is shown. (b) For the electrostatic potential in the far region (where $\rho \gg \rho_{0}$ ), the wedge and the original line charge can be replaced with the dominant term of (15), which is proportional to the fractional $\pi / \beta$ derivative of the line monopole. This fractional $\pi / \beta$ pole gives rise to a potential which is similar to the one shown in Fig. 2 . Here, the wedge angle is chosen to be $\beta=5 \pi / 4$. This results in $\alpha=\pi / \beta=0.8$ for which the contour plot is given in Fig. 2(b). The next higher-order term belongs to the first antisymmetric term in (15) with fractional $(\alpha-1)$ th-order derivative of a line dipole. For this term $\alpha=2 \pi / \beta=1.6$, and the contour plot for the potential of this term is shown in Fig. 3(b). Note that in both contour plots, the potential is zero at $\varphi=3 \pi / 8$ and $13 \pi / 8$ which correspond to the wedge surfaces.

which is effectively the potential of a 2-D line dipole with lines parallel with the $z$ axis located at the origin, and with the vector of the dipole $\boldsymbol{p}=2 Q_{l} \rho_{0} \cos \left(\varphi_{0}\right) \hat{\boldsymbol{x}}$ ( $\hat{\boldsymbol{x}}$ being the unit vector along the $x$ axis). Equation (17) for this case $(\beta=\pi)$ can be written as

$$
\begin{gathered}
Q_{\text {equiv }}(x, y)_{\beta=\pi} \approx-2 \rho_{0} \cos \left(\varphi_{0}\right)-\infty D_{x}^{1}\left[Q_{l} \delta(x) \delta(y)\right] \\
=2 Q_{l} \rho_{0} \cos \left(\varphi_{0}\right)\left[-\delta^{(1)}(x) \delta(y)\right] \\
\quad \text { for } \rho \gg \rho_{0} \\
\quad \text { and } \frac{\pi}{2}<\varphi_{0}<\frac{3 \pi}{2}
\end{gathered}
$$

This is, indeed, the volume charge density of a line dipole located at the origin with the same dipole vector $p=2 Q_{l} \rho_{0} \cos \left(\varphi_{0}\right) \hat{x}$ as mentioned before (note that $\pi / 2<\varphi_{0}<3 \pi / 2$ ). From the physical point of view it is clear that for this case, where the original line charge is parallel with the flat perfectly conducting interface at the $y-z$ plane, there is an image line charge with charge density per unit length $-Q_{l}$ located at $\left(\rho_{0}, \pi-\varphi_{0}\right)$ and parallel with the original line charge, and this image line charge $\left(-Q_{l}\right)$ and the original line charge $\left(Q_{l}\right)$ together form a line dipole which provides the dominant term of the potential in the far region, 
as given in (18). The dipole moment (per unit length of $z$ ) of this line charge is given by $\boldsymbol{p}=2 Q_{l} \rho_{0} \cos \left(\varphi_{0}\right) \hat{\boldsymbol{x}}$ which supports our findings above.

For the case where $\beta=\pi / 2$, we have a perfectly conducting $90^{\circ}$ corner. The electrostatic potential for $\rho \gg \rho_{0}$ is given by

$$
\begin{aligned}
\Phi_{l w}(\rho, \varphi)_{\beta=\pi / 2} \approx Q_{l} \rho_{0}^{2} \cos \left(2 \varphi_{0}\right) \frac{\cos (2 \varphi)}{\pi \varepsilon \rho^{2}} \\
\text { for } \rho \gg \rho_{0} \\
\text { and } \frac{3 \pi}{4}<\varphi_{0}, \varphi<\frac{5 \pi}{4} .
\end{aligned}
$$

It can be easily shown that this potential is the same as the potential of 2-D electrostatic line quadrupole $\underline{\underline{Q}}=$ $4 Q_{l} \rho_{0}^{2} \cos \left(2 \varphi_{0}\right) \hat{\boldsymbol{x}} \hat{\boldsymbol{x}}$. The charge from (17) for this case is expressed as

$$
\begin{aligned}
Q_{\text {equiv }}(x, y)_{\beta=\pi / 2} & \approx 2 \rho_{0}^{2} \cos \left(2 \varphi_{0}\right)_{-\infty} D_{x}^{2}[\delta(x) \delta(y)] \\
& =4 Q_{l} \rho_{0}^{2} \cos \left(2 \varphi_{0}\right)\left[\frac{1}{2} \delta^{(2)}(x) \delta(y)\right]
\end{aligned}
$$

The expression in the square brackets above which involves second derivative of $\delta(x)$, presents a volume charge density of a 2-D quadrupole with quadrupole moment $\underline{\underline{\boldsymbol{Q}}}=$ $4 Q_{l} \rho_{0}^{2} \cos \left(2 \varphi_{0}\right) \hat{\boldsymbol{x}} \hat{\boldsymbol{x}}$ which is the same as the line quadrupole generating the potential in (20). Therefore, the equivalent charge is consistent with the potential in the far region. To explain this case physically, we recall that for a 2-D $90^{\circ}$ corner with a line charge parallel with the edge of the corner, there exist three discrete image line charges. For the sake of simplicity in discussion here, let us assume that the original line charge is in the $x-z$ plane at the location $\left(\rho_{0}, \varphi_{0}=\pi\right)$. The three image line charges are, therefore, given as $+Q_{l}$ at $\left(\rho_{0}, 0\right)$, $-Q_{l}$ at $\left(\rho_{0}, \pi / 2\right)$, and $-Q_{l}$ at $\left(\rho_{0}, 3 \pi / 2\right)$. As seen from far, these three image line charges, together with the original line charge, form a 2-D quadrupole with quadrupole moment $\underline{\underline{Q}}=$ $2 Q_{l} \rho_{0}^{2} \cos \left(2 \varphi_{0}\right) \hat{\boldsymbol{x}} \hat{\boldsymbol{x}}-2 Q_{l} \rho_{0}^{2} \cos \left(2 \varphi_{0}\right) \hat{\boldsymbol{y}} \hat{\boldsymbol{y}}=2 Q_{l} \rho_{0}^{2}(\hat{\boldsymbol{x}} \hat{\boldsymbol{x}}-\hat{\overline{\hat{\boldsymbol{y}}}} \hat{\boldsymbol{y}})$. However, it can be easily shown that electrostatic potential due to $\underline{\underline{Q}}_{y y}$ (yy component of $\underline{\underline{Q}}$ ) is the same (but with the opposite sign) as that of the $\underline{\underline{Q}}_{x x}(x x$ component of $\underline{\underline{Q}})$. Therefore, the quadrupole moment $\underline{\underline{Q}}=4 Q_{l} \rho_{0}^{2} \cos \left(2 \varphi_{0}\right) \hat{\boldsymbol{x}} \hat{\boldsymbol{x}}$ produces the same far-zone potential as $\underline{\underline{\underline{Q}}}=2 Q_{l} \rho_{0}^{2} \cos \left(2 \varphi_{0}\right) \hat{\boldsymbol{x}} \hat{\boldsymbol{x}}-$ $2 Q_{l} \rho_{0}^{2} \cos \left(2 \varphi_{0}\right) \hat{\boldsymbol{y}} \hat{\boldsymbol{y}}$. This demonstrates that in this case, the charge distribution expressed in (21) is effectively consistent with the well-known discrete images, and it provides the same distant electrostatic potential for the 2-D $90^{\circ}$ corner. For other values of $\beta$ for which $\pi / \beta$ becomes an integer, one can show that the charge distribution (17) would similarly yield an electrostatic potential that is the same as the distant potential (i.e., where $\rho \gg \rho_{0}$ ) due to the discrete image line charges together with the original line charge.

Some of the salient features of the equivalent charges described in (15) (or (17) for the far observation points) should be noted.

1) As can be seen from (17) and from examples shown in Figs. 2 and 3, the fractional $\pi / \beta$ derivatives of the Dirac delta function give rise to singularities at $x=0$. For the observer in region $\rho \gg \rho_{0}$, the upward (positive) edge of this singularity may effectively appear as the original line charge, and the rest of the equivalent charge for $x>0$ can be viewed as the distributed "image" charges. For example, when $\beta=\pi$ for the conducting flat plate, the positive part of $\delta^{(1)}(x) \delta(y)$ can be regarded as the original line charge, and the negative part plays the role of the image of line charge.

2) For values of $\beta$ where $\pi / \beta$ is an integer, the order of derivatives involved in the definition of equivalent charge distribution becomes integer, thus yielding a conventional differentiation of Dirac delta function. This is consistent with the fact that for these values of $\beta$, discrete (localized) image line charges can be introduced. When $\pi / \beta$ is not an integer, then fractional derivatives of Dirac delta function are employed which yield distributed (nonlocalized) charge densities for the equivalent charges. So, effectively, as one gradually changes the angle of the wedge, the equivalent charge (15) or (17) "evolves" between discrete (localized) and distributed (nonlocalized) charge densities, continually "filling the gap" between the discrete cases of localized image line charges as line dipole, line quadrupole, line octupole, etc. Such an "intermediate" charge distribution was denoted as "fractional-order" poles. That is why we name this technique the "fractional" image method.

3) If the observer is not in the far region (but still $\rho>\rho_{0}$ ), higher-order terms given in (15) should also be included for the equivalent charge distribution. In this case, higher-order fractional derivatives become involved.

4) As can be easily seen from (15), there are two sets of terms in that equation: one set which includes the terms with $\cos \left\{[(2 n+1) \pi / \beta]\left(\pi-\varphi_{0}\right)\right\}$, and the other which has the terms with $\sin \left\{[(2 n+2) \pi / \beta]\left(\pi-\varphi_{0}\right)\right\}$. Physically, these sets correspond to the symmetric and antisymmetric parts of the source, respectively. In other words, we can consider the wedge problem with the line charge $Q_{l}$ at $\left(\rho_{0}, \varphi_{0}\right)$ as superposition of two subproblems: the symmetric part where there are two line charges of $Q_{l} / 2$ located at $\left(\rho_{0}, \varphi_{0}\right)$ and $\left(\rho_{0}, 2 \pi-\right.$ $\left.\varphi_{0}\right)$, and the antisymmetric part where there are two line charges $Q_{l} / 2$ and $-Q_{l} / 2$ located at $\left(\rho_{0}, \varphi_{0}\right)$ and $\left(\rho_{0}, 2 \pi-\varphi_{0}\right)$, respectively. The terms with $\cos \{[(2 n+$ 1) $\left.\pi / \beta]\left(\pi-\varphi_{0}\right)\right\}$ provide the equivalent charge for the symmetric part, and the terms with $\sin \{[(2 n+$ $\left.2) \pi / \beta]\left(\pi-\varphi_{0}\right)\right\}$ give the equivalent charge for the antisymmetric part. This is also supported by the fact that symmetric part involves fractional differentiation of line monopole, while antisymmetric part deals with fractional derivatives of line dipole.

5) Although our discussion has been aimed at observation points in region $\rho>\rho_{0}$, it can be shown that when $\beta>\pi$ for the region close to the edge of the wedge where $\rho \ll \rho_{0}$, an equivalent charge distribution similar to (17) can be obtained provided that terms involving 
$\pi / \beta$ in (17) be replaced by $-\pi / \beta$. Thus, we get

$$
\begin{aligned}
Q_{\text {equiv }}(x, y) \approx & \frac{-2 \pi \rho_{0}^{-\pi / \beta} \cos \left[\frac{\pi}{\beta}\left(\pi-\varphi_{0}\right)\right]}{\beta \Gamma\left(1-\frac{\pi}{\beta}\right)} \\
& -_{-\infty} D_{x}^{-\pi / \beta}\left[Q_{l} \delta(x) \delta(y)\right] \\
& \text { for } \beta>\pi \\
& \text { and } \rho \ll \rho_{0}
\end{aligned}
$$

as the equivalent charge which, in the absence of the wedge and the original line charge, would provide the same potential for the observation points in the region $\rho \ll \rho_{0}$. It is interesting to note that this equivalent charge is expressed in terms of fractional integral of the line monopole.

Given the points made in the foregoing analysis and discussion, it is clear that for a perfectly conducting wedge with arbitrary angle $\beta$, the general charge distributions introduced in this section can be considered as "image" charges, and they provide the equivalent electrostatic wedge potential in absence of the wedge and its original line charge.

\section{EXTENSION TO THE CASE OF PERFECTLY CONDUCTING CONES WTTH ARBITRARY ANGLE}

The concept of "fractional" image method, introduced in the previous section, can be extended to the 3-D case of perfectly conducting cone with arbitrary angle. The mathematical details are somewhat similar to what was described in Section II, and thus are not repeated here in detail.

Let us consider a perfectly conducting cone in the Cartesian $(x, y, z)$ and spherical $(r, \theta, \varphi)$ coordinate systems. As shown in Fig. 5, the $z$ axis is taken to be the symmetry axis of the cone, and the cone angle is $\beta$. The cone region $0<\theta<\beta$ is perfectly conducting and the rest is taken to be a homogeneous isotropic medium of permittivity $\epsilon$. A point charge $Q_{0}$ is located at an arbitrary point $\left(r_{0}, \theta_{0}, \varphi_{0}\right)$ where $\beta<\theta_{0}<\pi$. For observation points with $r>r_{0}$, the electrostatic potential $\Phi_{p c}$ of this point charge in front of the cone can be written using spherical harmonics [17], as shown in (23) at the bottom of this page, where $P_{\nu_{m, n}}^{m}[\cdot]$ are associated Legendre functions of the first kind of degree $\nu_{m, n}$ and order $m$, the degrees $\nu_{m, 1}, \nu_{m, 2}, \nu_{m, 3}, \cdots$ are the roots of the equation $P_{\nu_{m, n}}^{m}[\cos (\pi-\beta)]=0$, the value of $I_{m, \nu_{n}}$ is defined as $I_{m, \nu_{m, n}} \equiv \int_{\cos (\pi-\beta)}^{1}\left\{P_{\nu_{m, n}}^{m}[\cos (\pi-\theta)]\right\}^{2} d[\cos (\pi-\theta)]$, and $\delta_{0 m}$ is the Kronecker delta. Without the cone, the potential

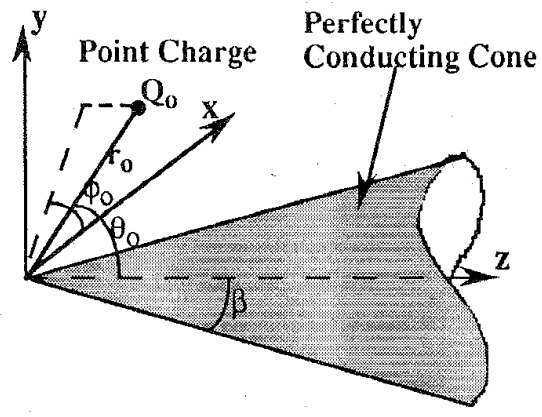

Fig. 5. A perfectly conducting cone with angle $\beta$. A single static point charge $Q_{0}$ is located at $\left(r_{0}, \theta_{0}, \varphi_{0}\right)$.

of the same single-point charge located at the same point is given, as shown in (24) at the bottom of this page. For $r<r_{0}$, the role of $r$ and $r_{0}$ should be interchanged in the above expressions. As shown by Maxwell, the spherical harmonics $P_{n}^{m}[\cos (\theta)]\left\{\begin{array}{l}\cos (m \varphi) \\ \sin (m \varphi)\end{array}\right.$ can be written in terms of spatial derivatives of $r^{-1}$ [8, pp. 207-214]. Therefore, for $r>r_{0}, \Phi_{p o}$ given in (24) can be written as

$$
\begin{aligned}
\Phi_{p o}= & \sum_{m=0}^{\infty} \sum_{n=m}^{\infty} \frac{2^{m} Q_{0} r_{0}^{n} P_{n}^{m}\left[\cos \left(\pi-\theta_{0}\right)\right]}{(n+m) !} \\
& \cdot\left[\cos \left(m \varphi_{0}\right) \frac{\partial^{n-m}}{\partial z^{n-m}} \frac{\partial_{c}^{(m)}}{\partial(x, y)^{(m)}}\right. \\
& \left.+\sin \left(m \varphi_{0}\right) \frac{\partial^{n-m}}{\partial z^{n-m}} \frac{\partial_{s}^{(m)}}{\partial(x, y)^{(m)}}\right] \\
& \cdot\left(\frac{1}{4 \pi \varepsilon r}\right) . \text { for } r>r_{0} .
\end{aligned}
$$

Here, the notations $\partial_{c}^{(m)} / \partial(x, y)^{(m)}$ and $\partial_{s}^{(m)} / \partial(x, y)^{(m)}$ represent certain spatial differentiation in the $x-y$ plane along $m$ directions oriented at angular intervals of $\pi / m[8$, p. 210$]$ and $[27, \mathrm{pp} .149-152]$. These differentiations in the $x-y$ plane was used by Maxwell to generate the $\cos (m \varphi) P_{m}^{m}(\cos \theta)$ and $\sin (m \varphi) P_{m}^{m}(\cos \theta)$ parts of the spherical harmonics. ${ }^{7}$

For the cone problem, however, using mathematical steps similar to what we showed in Section II, we can show that the expression for potential $\Phi_{p c}$ shown in (23) can be written in

\footnotetext{
${ }^{7}$ The symbols used by Maxwell in his treatise [8, p. 211] are $D_{c}^{(\sigma)}$. and $D_{s}^{(\sigma)}$ where his $(\sigma)$ is our $m$ here. However, here we use a different symbol to avoid confusion with the symbol ${ }_{a} D_{z}^{\alpha}$ which we use for the fractional derivatives.
}

$$
\begin{aligned}
\Phi_{p c} & =\sum_{m=0}^{\infty} \sum_{\nu_{m, n}} \frac{Q_{0} r_{0}^{\nu_{m, n}} \cos \left[m\left(\varphi-\varphi_{0}\right)\right] P_{\nu_{m, n}}^{m}\left[\cos \left(\pi-\theta_{0}\right)\right] P_{\nu_{m, n}}^{m}[\cos (\pi-\theta)]}{\left(1+\delta_{0 m}\right)\left(2 \nu_{m, n}+1\right) \pi \varepsilon I_{m, \nu_{m, n}} r^{\nu_{m, n}+1}} \text { for } r>r_{0} \\
\Phi_{p o} & =\sum_{m=0}^{\infty} \sum_{n=m}^{\infty} \frac{Q_{0} r_{0}^{n} \cos \left[m\left(\varphi-\varphi_{0}\right)\right] P_{n}^{m}\left[\cos \left(\pi-\theta_{0}\right)\right] P_{n}^{m}[\cos (\pi-\theta)]}{\left(1+\delta_{0 m}\right) 2 \pi \varepsilon \frac{(n+m) !}{(n-m) !} r^{n+1}} \text { for } r>r_{0}
\end{aligned}
$$


terms of the fractional derivatives of $1 /(4 \pi \epsilon r)$ as follows:

$$
\begin{aligned}
\Phi_{p c}= & \sum_{m=0}^{\infty} \sum_{\nu_{m, n}} \frac{2^{m+1} Q_{0} r_{0}^{\nu_{m, n}} P_{\nu_{m, n}}^{m}\left[\cos \left(\pi-\theta_{0}\right)\right]}{\left(2 \nu_{m, n}+1\right) I_{m, \nu_{m}, n} \Gamma\left(\nu_{m, n}-m+1\right)} \\
& {\left[\cos \left(m \varphi_{0}\right)_{-\infty} D_{z}^{\nu_{m, n}-m} \frac{\partial_{c}^{(m)}}{\partial(x, y)^{(m)}}\right.} \\
& \left.+\sin \left(m \varphi_{0}\right)_{-\infty} D_{z}^{\nu_{m, n}-m} \frac{\partial_{s}^{(m)}}{\partial(x, y)^{(m)}}\right]\left(\frac{1}{4 \pi \varepsilon r}\right) \\
& \text { for } \quad r>r_{0} .
\end{aligned}
$$

Therefore, instead of conventional differentiation used to describe the potential of a single charge (in the absence of the cone), as shown in (25), fractional differentiation (with respect to $z$ ) should be used in (26). The above expression shows that for the potential in region $r>r_{0}$, we can introduce an equivalent charge distribution involving fractional derivatives of point charge which would produce a potential similar to, the potential of the point charge in front of the perfectly conducting cone. This equivalent charge distribution can be written as

$$
\begin{aligned}
Q_{\text {equiv }}= & \sum_{m=0}^{\infty} \sum_{\nu_{m, n}} \frac{2^{m+1} r_{0}^{\nu_{m, n}} P_{\nu_{m, n}}^{m}\left[\cos \left(\pi-\theta_{0}\right)\right]}{\left(2 \nu_{m, n}+1\right) I_{m, \nu_{m, n}} \Gamma\left(\nu_{m, n}-m+1\right)} \\
& \cdot\left[\cos \left(m \varphi_{0}\right)_{-\infty} D_{z}^{\nu_{m, n}-m} \frac{\partial_{c}^{(m)}}{\partial(x, y)^{(m)}}\right. \\
& \left.\quad+\sin \left(m \varphi_{0}\right)_{-\infty} D_{z}^{\nu_{m, n}-m} \frac{\partial_{s}^{(m)}}{\partial(x, y)^{(m)}}\right] \\
& \cdot\left[Q_{0} \delta(x) \delta(y) \delta(z)\right] \text { for } r>r_{0} .
\end{aligned}
$$

So, if we remove the original point charge and the conducting cone and replace them with the above equivalent charge, we would get the same cone potential distribution for the observation points in the region $r>r_{0}$. Thus, for $r>r_{0}$ this represents an equivalent "image" charge for the problem of perfectly conducting cone with a point charge in its vicinity.

It is of interest to consider the case of the distant observers where $r \gg r_{0}$. In this case, the dominant term of the potential will have $m=0$ and the smallest $\nu_{0, n}$. The charge distribution given in (27) for this case is given as

$$
\begin{aligned}
Q_{\text {equiv }} \approx & \frac{2 r_{0}^{\nu_{0,1}} P_{\nu_{0,1}}\left[\cos \left(\pi-\theta_{0}\right)\right]}{\left(2 \nu_{0,1}+1\right) I_{0, \nu_{0,1}} \Gamma\left(\nu_{0,1}+1\right)} \\
& \cdot-\infty D_{z}^{\nu_{0}, 1}\left[Q_{0} \delta(x) \delta(y) \delta(z)\right] \\
= & \frac{2 Q_{0} r_{0}^{\nu_{0,1}} P_{\nu_{0,1}}\left[\cos \left(\pi-\theta_{0}\right)\right]}{\left(2 \nu_{0,1}+1\right) I_{0, \nu_{0,1}} \Gamma\left(\nu_{0,1}+1\right)} \frac{\partial^{p}}{\partial x^{p}} \\
& \cdot \begin{cases}\delta(x) \delta(y) \frac{1}{\Gamma\left[p-\nu_{0,1}\right]} z^{-\nu_{0,1}+p-1} & \text { for } z>0 \\
0 & \text { for } z<0\end{cases} \\
& \quad \text { for } r \gg r_{0}
\end{aligned}
$$

where $\nu_{0,1}$ can be a noninteger positive number and, as in (17), the integer $p$ is chosen such that $\nu_{0,1}-p$ becomes negative (see footnote 5). For noninteger values of $\nu_{0,1}$, this 3-D chargedensity distribution is a (nonlocalized) line-charge distribution along the $z$ axis for $z>0$ with volume charge density given

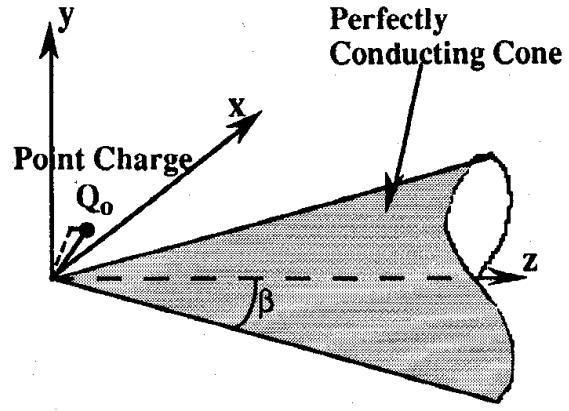

(a)

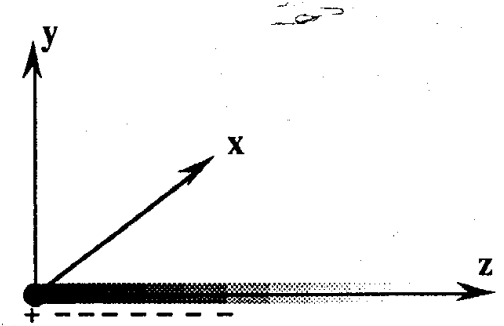

(b)

Fig. 6. (a) Illustration of a perfectly conducting cone with the cone angle $\beta$. A single static point charge $Q_{0}$ is in the vicinity of this cone. For the electrostatic potential in the far region (where $r \gg r_{0}$ ), the cone and the original point charge $Q_{0}$ can be replaced with the equivalent charge shown in (28). (b) The sketch of this charge, which is proportional to the fractional $\nu_{0,1}$ derivative of a point monopole is shown. The noninteger $\nu_{0,1}$ is the smallest root for $P_{\nu_{0}}[\cos (\pi-\beta)]=0$. This $3-\mathrm{D}$ charge-density distribution is a nonlocalized line charge distribution along the $z$ axis for $z>0$, with charge density given in $(28)$.

in the above equation and with a singularity at $z=0$. The sketch of such a charge distribution is given in Fig. 6(b). If we consider the special case of $\beta=\pi / 2$ for which the cone becomes a flat plate, the roots of $P_{\nu_{0}, n}[\cos (\pi-\beta)]=0$ will be $\nu_{0,1}=1, \nu_{0,2}=3, \nu_{0,3}=5, \cdots$. Thus, the charge distribution given in (28) for $\nu_{0,1}=1$ has the form $Q_{\text {equiv }} \approx$ $-2 r_{0} \cos \left(\pi-\theta_{0}\right)\left[-Q_{0} \delta(x) \delta(y) \delta^{(1)}(z)\right]$ which represents a point dipole with dipole moment $\boldsymbol{p}=-2 Q_{0} r_{0} \cos \left(\pi-\theta_{0}\right) \hat{z}$ at the origin. This is consistent with the conventional method of images when a point charge is located in front of a perfectly conducting plate. Most of the salient features of the "fractional" image method for the cone are similar to those described for the wedge.

\section{SUMMARY}

In this paper, we have introduced and described, in detail, the concept of "fractional" image methods for the electrostatic problem involving the perfectly conducting wedge and cone. Using fractional calculus and the concept of "fractional-order" poles, we have shown that the electrostatic potentials in front of perfectly conducting wedges and cones can be expressed equivalently as the potentials of sets of equivalent charges that have the form of fractional-order poles. The "order" of these poles depends on the wedge angle (for the 2-D wedge problem) and on the cone angle (for the 3-D cone problem). We have also shown that the potential of these general "fractional images" approach that of the well known discrete images for the special cases. 
These fractional "image" charges may have applications in the analysis of electrostatic problems involving these structures. For example, one may speculate that in numerical evaluation of fields in the presence of sharp edges and tips, the knowledge of the form of these equivalent "image" charges may become useful. Extension of this method to the electrostatic 2-D dielectric wedge is currently under study by the author.

\section{REFERENCES}

[1] N. Engheta, "On fractional calculus and fractional multipoles in electromagnetism," IEEE Trans. Antennas Propagat., vol. 44, pp. 554-566, Apr. 1996.

[2] _. _ "Fractional derivatives, fractional integrals, and electrostatic image methods," presented at URSI'95 IEEE Antennas Propagat. SOC. Int. Symp./USNC-URSI Radio Sci. Meet., Newport Beach, CA, June 18-23, 1995 (1-page abstract in URSI Dig., p. 245).

[3] _ "A note on fractional calculus and the image method for dielectric spheres," J. Electromagn. Waves Applicat., vol. 9, no. 9, 1179-1188, 1995

[4] "Use of fractional integration to propose some 'fractional'. solutions for the scalar Helmholtz equation," in Progress in Electromagnetics Research (PIER), J. A. Kong, Ed. Cambridge, MA: EMW Pub., 1996, vol. 12 , pp. $107-132$.

[5] R. R. Nigmatulin, "On the theory of relaxation for systems with 'remnant' memory," Phys. Status Solidi (B), vol. 124, no. 1, pp. 389-393, July 1984

[6] A. Le Méhauté, F. Héliodore, and V. Dionnet, "Overview of electrical processes in fractal geometry: From electrodynamic relaxation to superconductivity," Proc. IEEE, vol. 81, pp. 1500-1510, Oct. 1993.

[7] A. Le Méhauté, F. Héliodore, D. Cottevieille, and F. Latreille, "Introduction to wave phenomena and uncertainty in a fractal space-I," Chaos, Solitons, Fractals, vol. 4, no. 3, pp. 389-402, 1994.

[8] J. C. Maxwell, Treatise on Electricity and Magnetism. New York: Dover, 1954, vol. 1 (reprint).

[9] W. R. Smythe, Static and Dynamic Electricity, 3rd ed. New York: McGraw-Hill, 1968.

[10] I. V. Lindell, J. C.-E. Sten, and R. E. Kleinman, "Low-frequency image theory for the dielectric sphere," J. Electromagn. Waves Applicat., vol. 8, no. 3, pp. $295-313,1994$.
[11] I. V. Lindell, "Electrostatic image theory for the dielectric sphere," Radio Sci., vol. 27, no. 1, pp. 1-8, Jan./Feb. 1992.

[12] K. I. Nikoskinen and I. V. Lindell, "Image solution for Poisson's equation in wedge geometry," IEEE Trans. Antennas Propagat., vol. 43, pp. 179-187, Feb. 1995.

[13] M. E. Ermutlu, I. V. Lindell, and K. I. Nikoskinen, "Two-dimensional image theory for the conducting wedge," J. Electromagn. Waves Applicat., vol. 7, no. 7, pp. 971-986, 1993.

[14] I. V. Lindell, M. E. Ermutlu, and K. I. Nikoskinen, “Two-dimensional theory for the conducting half plane," J. Electromagn. Waves Applicat., vol. 7 , no. 2, pp. 179-196, 1993.

[15] K. I. Nikoskinen, I. V. Lindell, and M. E. Ermutlu, "Transient analysis of conducting half-plane problem with noncausal scattering image," Microwave Opt. Tech. Lett., vol. 7, nio. 1, pp. 31-35, 1994.

[16] K. I. Nikoskinen, M. E. Ermutlu, and I. V. Lindell, "Transient image theory for 2-D and 3-D conducting wedge problems," IEEE Trans. Antennas Propagat., vol. 42, pp. 1515-1520, Nov. 1994.

[17] J. D. Jackson, Classical Electrodynamics, 2nd ed. New York: Wiley, 1975.

[18] I. M. Gel'fand and G. E. Shilov, Generalized Functions. New York: Academic, 1964-1968 (5 vols.).

[19] L. Schwartz, Théorie des Distributions. Paris, France: Hermann, 1966.

[20] C. H. Papas, Theory of Electromagnetic Wave Propagation. New York: Dover, 1988; McGraw-Hill, 1965 (unabridged reprint).

[21] J. Van Bladel, "The multipole expansion revisited," Archiv fiir Elektron. Übertragungstech., vol. 31 , pp, 407-411, 1977.

[22] V. Namias, "Application of the Dirac delta function to electric charge and multipole distributions," Amer. J. Phys., vol. 45, no. 7, pp. 624-630, July 1977 .

[23] C. A. Kocher, "Point-multipole expansion for charge and current distributions," Amer. J. Phys., vol. 46, no. 5, pp. 578-579, May 1978.

[24] K. B. Oldham and J. Spanier, The Fractional Calculus. New York: Academic, 1974.

[25] H. T. Davis, The Theory of Linear Operators. Bloomington, $\mathbb{N}$ : Principia Press, 1936, pp. 64-75, 276-292.

[26] S. G. Samko, A. A. Kilbas, and O. I. Marichev, Fractional Integrals and Derivatives, Theory, and Applications. Langhorne, PA: Gordon Breach Sci. Publ., 1993 (trans. from Russian by N. Tekhnika, Minsk, 1987).

[27] A. Sommerfeld, Partial Differential Equations in Physics Lectures on Theoretical Physics. New York: Academic, 1949, vol. VI.

Nader Engheta (S'81-M'82-SM'89-F'96), for photograph and biography, see p. 566 of the April 1996 issue of this TRANSACTrons. 